\title{
Experimental Study on In-situ Remediation of Compound Contaminated Sediment of a River in Shenzhen with Chemicals
}

\author{
Wang De-yong ${ }^{1,2,3, *}$, Wang Jing ${ }^{1,2,3}$, Zeng Qing-jun ${ }^{1,2}$, Deng Kai-fang ${ }^{1}$ \\ ${ }^{1}$ CCCC Fourth Harbor Engineering Institute Co.,Ltd,Guangzhou 510230,China; \\ ${ }^{2}$ Key Laboratory of Environmental Protection \& Safety of Communication Foundation Engineering,CCCC, Guangzhou 510230,China \\ ${ }^{3}$ Southern Marine Science and Engineering Guangdong Laboratory, Zhuhai 519082, China
}

\begin{abstract}
Aiming at the black and odor problem of the gushing water and sediment of a river in Shenzhen, based on the results of sampling and testing, we independently developed an in-situ oxidation remediation agent for polluted sediment composed of oxidants, flocculants and $\mathrm{pH}$ regulators, and carried out in-situ repair of chemical agents without and with overlying water indoor test to verify. The test and monitoring results show that the agent can not only significantly reduce the ammonia nitrogen and organic matter content in the bottom sludge, but also can better remove the ammonia nitrogen in the overlying black and odorous water. In order to get closer to the actual situation, using the reagents developed to repair contaminated sediments at a certain flow rate has also achieved good experimental results.
\end{abstract}

\section{Introduction}

The treatment of black and odorous water bodies is the focus of the prevention and control of urban water pollution in my country, and it has now entered the critical stage. The treatment of black and odorous water is not only the treatment of water problems, but also the treatment of the bottom sludge of the black and odorous water. Sediment repair technology is divided into two categories: in-situ repair and ex-situ repair ${ }^{[1]}$. Due to ex-situ remediation, there are problems such as difficulty in stacking and disposal of bottom mud, troublesome and expensive transportation, and easy to cause secondary pollution. Therefore, the in-situ remediation technology of contaminated sediment has received more and more attention. At present, in-situ remediation includes physical, chemical, bioremediation technology and a combination of multiple techniques ${ }^{[2]}$.

Physical remediation ${ }^{[3]}$ focuses on in-situ coverage, stabilizing sediments to prevent resuspension and the migration of pollutants. It is effective and low-cost, but it has defects such as reducing water body capacity, changing river courses and lake bottom slopes. Bioremediation technology ${ }^{[4]}$ uses the life activities of aquatic plants and microorganisms to adsorb, transfer, transform and degrade pollutants in water bodies, thereby purifying the water body and rebuilding the water ecosystem. The project cost is relatively low, but a single Biological treatment technology cannot fully solve the problem of river and lake sediment pollution due to its strong pertinence, and has poor treatment effects on heavy metals, phosphorus and other pollutants. Chemical remediation technology ${ }^{[5]}$ eliminates pollutants in the bottom sludge through chemical reactions or changes the properties of the original pollutants. It is generally suitable for direct contact with water bodies of non-human bodies with heavy sediment pollution, and has a better effect on sediments contaminated by heavy metals. This method has low cost and quick effect, and is currently widely used. Of course, the dosage of medicament should be strictly controlled to avoid secondary pollution and potential public safety hazards.

A river near Shenzhen Bao'an Airport has a black and odorous water body, and the black bottom mud has turned up, which seriously affects the water quality and perception of the water body. In order to repair the black odor sediment in the river in situ quickly, based on the project, the sediment from the river was sampled and analyzed, and then the chemical restoration agent was developed according to the test results, and then the laboratory test was carried out.

\section{Sediment sampling and testing}

A columnar mud dredger was used to sample at the bottom of the supporting project, and the bottom sludge from 0 to $50 \mathrm{~cm}$ in the surface layer was collected. Samples were taken 3 times at each sampling point. The field sampling is shown in Figure 1. After removing the debris, mix the sample evenly, cover it with water for storage, and seal it for packaging. Immediately after sampling, transport them back to the laboratory for pretreatment. 


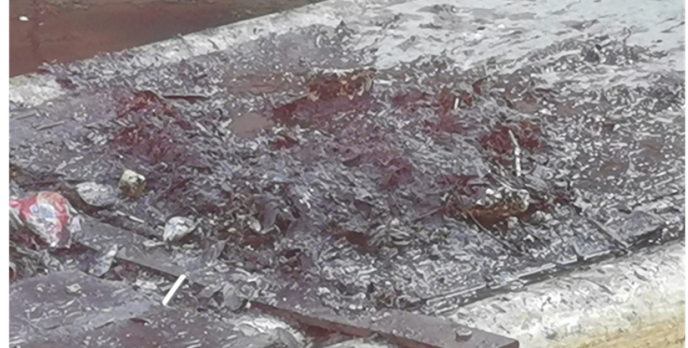

Fig.1 On-site sampling of sediments from a river in Shenzhen

Conduct physical and mechanical property tests and chemical tests for contaminated sediment sampling. Understand the composition, physical properties and basic chemical properties of polluted sediments, analyze the distribution characteristics of composite pollutants in sediments, and provide a scientific reference for the proposal of in-situ treatment technology for complex pollution of river sludge. Physical indicators include: natural moisture content, transparency, chroma; chemical indicators include: dissolved oxygen DO, odor threshold, $\mathrm{pH}$, organic matter, ammonia nitrogen and nitrate nitrogen, total organic carbon, heavy metals (As, Cd, $\mathrm{Zn}$, $\mathrm{Ni}, \mathrm{Cr}, \mathrm{Cu}, \mathrm{Hg}, \mathrm{Pb}$ solid phase total). The analysis method of each index adopts the national standard method or the environmental protection industry standard method.

\section{Characteristic analysis of sediment pollution}

The bottom sludge and overlying water were sampled in two typical rivers that rely on the project, and their ammonia nitrogen, total phosphorus, COD, $\mathrm{PH}$ and organic matter were tested. The test results are shown in Tables 1 to 2 below.

Tab.1 Sample test results

\begin{tabular}{|c|c|c|c|c|c|}
\hline object & $\begin{array}{c}\mathrm{NH}_{3}-\mathrm{N} \\
(\mathrm{mg} / \mathrm{L})\end{array}$ & $\begin{array}{c}\mathrm{TP} \\
(\mathrm{mg} / \mathrm{L})\end{array}$ & $\begin{array}{c}\mathrm{OM} \\
(\mathrm{g} / \mathrm{kg})\end{array}$ & $\begin{array}{c}\mathrm{COD} \\
(\mathrm{mg} / \mathrm{L})\end{array}$ & $\mathrm{PH}$ \\
\hline mud & 2.18 & 0.519 & 11.03 & $/$ & $/$ \\
\hline water & 8.07 & 1.18 & $/$ & 44.96 & 6.7 \\
\hline
\end{tabular}

Tab.2 Analysis results of heavy metals in bottom mud

\begin{tabular}{|c|c|c|c|c|c|}
\hline $\mathrm{Cr}$ & $\mathrm{Zn}$ & $\mathrm{Pb}$ & $\mathrm{Cu}$ & $\mathrm{Ni}$ & total PCB \\
\hline \multicolumn{5}{|c|}{$\mathrm{mg} / \mathrm{kg}$} \\
\hline 0.04 & 83.58 & 36.36 & 52.92 & 21.79 & 0.04 \\
\hline
\end{tabular}

The bottom sludge sediment is black in color, sparse and uniform in a gel state, and has a $\mathrm{pH}$ of about 6.5. A qualitative analysis of the complex organic components and heavy metal components of the sediments by gas chromatography-mass spectrometry and flame atomic absorption was used. The analysis results are summarized as follows.

By analyzing the heavy metal concentration levels of the main pollutants in the bottom sludge of rivers, and comparing them with local soil background soil samples, "Agricultural Land Soil Pollution Risk Management
Standards" and "Soil Heavy Metal Background Values", the preliminary results are analyzed as follows:The content of heavy metals $(\mathrm{Cd}, \mathrm{Pb}, \mathrm{Cu}, \mathrm{Ni}, \mathrm{Zn})$ in all landfills did not exceed the second-class land screening value of the soil pollution risk control standard for agricultural land. However, compared with the background value of heavy metals in the soil of Guangdong Province, the heavy metal content of $\mathrm{Cd}, \mathrm{Cu}$, and $\mathrm{Ni}$ in the sample is close to the background value, showing light pollution. Among them, the $\mathrm{Zn}$ concentration in the sample is much higher than the background value, which has a certain impact on the ecological environment and human health pollution effects. The content of heavy metals in the sediments of rivers is excessive, which has potential environmental risks.

By analyzing the concentration levels of main pollutants in the sediments of rivers, and comparing them with the "Agricultural Land Soil Pollution Risk Management and Control Standards", the preliminary results are analyzed as follows:The content of major organic pollutants such as polychlorinated biphenyls (PCBs) in the soil did not exceed the screening value of the second type of land used in the soil pollution risk control standard for agricultural land. It has potential environmental risks for its pollution effects on the ecological environment and human health.

\section{Research and development of sediment treatment agents and result analysis}

\subsection{Pharmaceutical R\&D and verification test}

In order to achieve the removal of organic matter in the bottom sludge, combined with engineering application and cost issues, the laboratory uses two kinds of drug A (calcium peroxide) and drug B (PFS, polyferric sulfate) for the treatment of black and odorous bottom sludge, supplemented by $\mathrm{pH}$ regulator $(\mathrm{NaOH})$ in real time.

In the experiment, $\operatorname{drug} \mathrm{A}, \operatorname{drug} \mathrm{B}, \operatorname{drug} \mathrm{A}+\mathrm{B}$ and $\mathrm{a}$ group of drug-free administration were added to $10 \mathrm{~g}$ of sediment in a mass ratio of 1:20 as a control experiment.

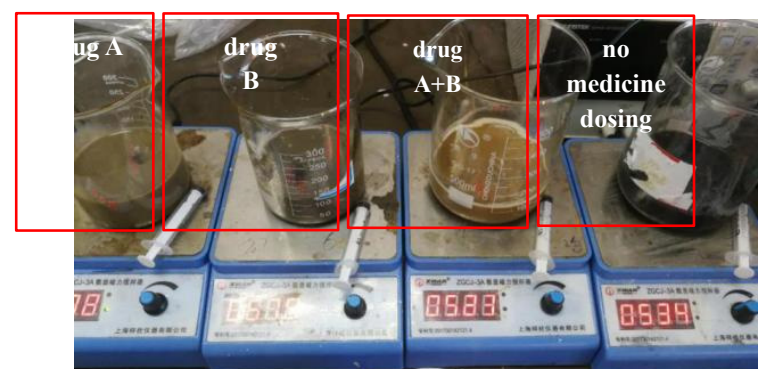

Fig.2 Effect comparison chart after reaction for 30 minutes

The above picture is a comparison of the color of the bottom sludge when the reaction time is 30 minutes. It can be seen that all three treatments have a significant effect on the decolorization of the bottom sludge. 


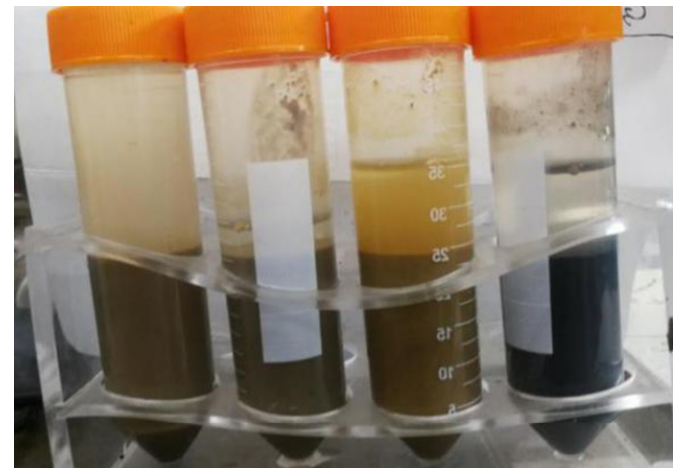

Fig.3 Effect comparison chart after $3 \mathrm{~d}$ reaction

After the dosing treatment, there was no obvious change in color after standing for three days. While the bottom sludge is decolorized, drug A has a significant effect on the removal of ammonia nitrogen in the overlying water, and it can quickly degrade the ammonia nitrogen in the overlying water under the condition of continuous stirring.

Next, we further optimized the dosage ratio. We selected the drug $\mathrm{A}+\mathrm{B}$, which had the best effect in the previous test, and selected four groups of $1 \%$ to $10 \%$ (B/A) for the experiment again. The results are as follows.

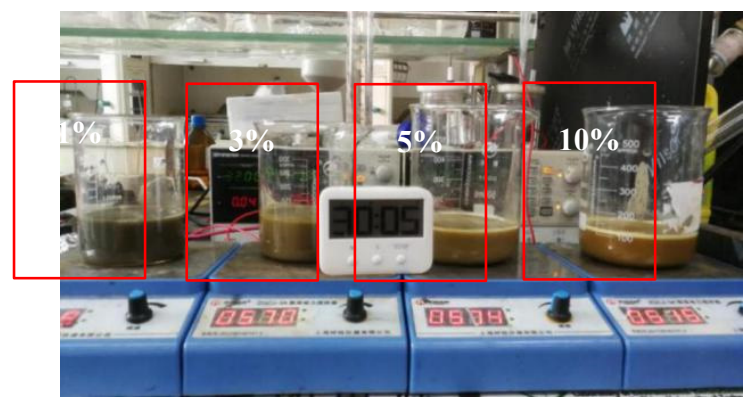

Fig.4 Effect after treatment with different dosage ratios

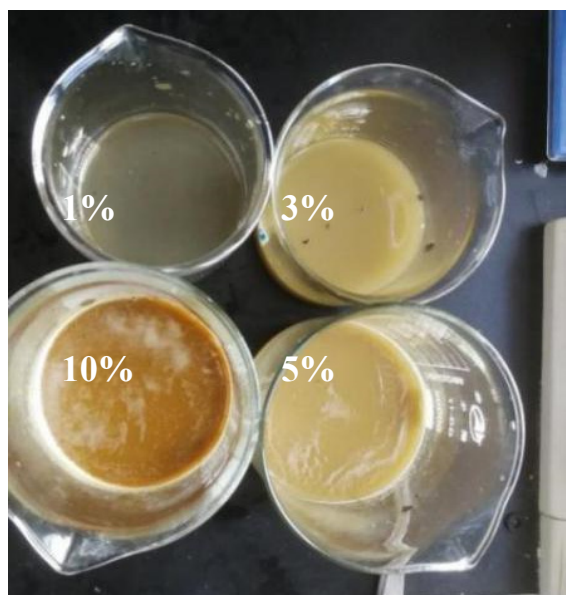

Fig.5 The effect of different mixing ratio reagents after treatment

\subsection{Test while water body is flowing}

In order to get closer to the actual working conditions of the river, the research and development agent was used to carry out the indoor test of the contaminated sediment remediation of the water body at a certain flow rate, as shown in Figure 5. It can be seen from the figure that the bottom mud before treatment in the measuring cup is black. Put it into the treatment device and pass water at a certain flow rate. After adding the medicine and stirring, the color of the bottom mud turns into a healthy khaki. It shows that the reagents developed can treat the polluted sediments of rivers with a certain flow rate.

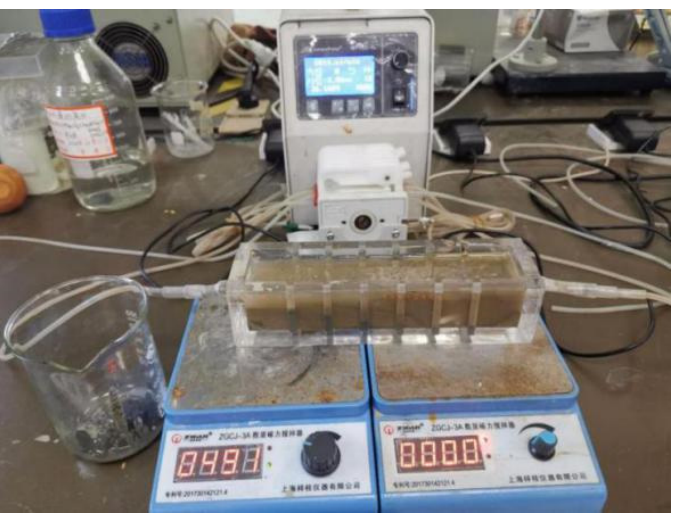

Fig.6 Indoor test for remediation of contaminated sediments at a certain flow

\subsection{Result analysis}

The test results of rapid treatment of sediments are shown in Table 3.

Tab.3 Test data result

\begin{tabular}{|c|c|c|c|c|}
\hline project & $\begin{array}{c}\text { untreated } \\
\text { bottom } \\
\text { sludge }\end{array}$ & $\begin{array}{c}\text { blank } \\
\text { control }\end{array}$ & drug A & $\begin{array}{c}\text { drug } \\
\mathrm{A}+\mathrm{B}\end{array}$ \\
\hline $\begin{array}{c}\text { ammonia } \\
\text { nitrogen } \\
(\mathrm{mg} / \mathrm{kg})\end{array}$ & 2181.3 & 1722.6 & 212.5 & 186.8 \\
\hline $\begin{array}{c}\text { organic } \\
\text { matter }(\mathrm{g} / \mathrm{kg})\end{array}$ & 11.03 & 8.00 & 5.97 & 5.90 \\
\hline
\end{tabular}

Drug A has a significant effect on the ammonia nitrogen and organic matter content of the bottom sludge, and also has a good effect on the removal of ammonia nitrogen in the overlying water.

Excessive drug A was added to the overlying water samples of the samples taken on site. The test results showed that drug A can remove more than $95 \%$ of the ammonia nitrogen $(3.96 \mathrm{mg} / \mathrm{L})$ in the water within 15 minutes.

Due to the certain difference between laboratory conditions and on-site environmental conditions, in order to further study the applicability and effectiveness of this technology, it is particularly necessary to conduct on-site comparison tests. This oxidant can be prepared at the project site, which can be a safety issue for oxidant transportation and storage.

\section{Conclusion}

Relying on a polluted river in Shenzhen, we have developed in-situ chemical agents for repairing the black 
and odorous bottom sludge in response to the black and odor problems of the bottom sludge and water bodies. Through laboratory experiments, the effect of sediment remediation agent on the release of sediment pollutants and the overlying water was explored, and the effect of this technology was analyzed. The main conclusions drawn are as follows:

(1) When chemical agents are used to repair the contaminated bottom sludge, the contaminated bottom sludge with calcium peroxide $\left(\mathrm{CaO}_{2}\right)$ is added to form a brown calcified layer, and polyferric sulfate (PFS) is added to form a flocculated layer on the surface of the bottom sludge to make the color clear. The effect of the compound agent combines the advantages of the two, forming a solidified layer, but also has the covering effect of the flocculating layer; the appropriate ratio of PFS and $\mathrm{CaO}_{2}$ is $5 \%$.

(2) The compound medicament can not only significantly reduce the ammonia nitrogen and organic matter content of the sediment, but also effectively remove the ammonia nitrogen in the overlying water.

(3) The reagent developed can not only treat the polluted sediment in still water, but also the polluted sediment in the river with a certain flow rate.

\section{References:}

1. Wang Chao, Chen Liang, Liao Si-hong. Research progress in mud in-situ repair technology. Green technology. 2014, (11): 165-166.

2. Xu Lian-feng, Deng Shao-long, Chen Ji-xin, Xia Zhong-wen, River sediment pollution and its control and restoration, Journal of Ecological Environment 2014, 23(10): 1708-1715.

3. Zhou Ying, Pan Gang, Chen Hao, Research on the effect of soil in-situ mulching on the remediation of sediments, Chinese Journal of Environmental Engineering, 2011, 5(11): 2459-2464.

4. Liu Yao-min, Wang Ru-song, Jiang Yao-ying, etc. The effect of in-situ biotechnology on the treatment of heavily polluted river sediments in cities, Acta Ecologica Sinica, 2013, 33(8): 2358-2364.

5. Niu Meiqing, Su Yugeng, Qiao Yongbo, Wu Renchao. Remediation effect of 4 kinds of chemical agents on black and smelly water bottom mud, Guangzhou Chemical Industry, 2019, 47(24): 48-50. 